\title{
Philonsorbonne
}

15 | 2021

Année 2020-2021

\section{Sade et la valorisation de l'indifférence : le paradoxe de la tolérance libertine}

Danilo BILATE

\section{(2) OpenEdition}

1 Journals

Édition électronique

URL : https://journals.openedition.org/philonsorbonne/1901

DOI : 10.4000/philonsorbonne.1901

ISSN : $2270-7336$

Éditeur

Publications de la Sorbonne

\section{Édition imprimée}

Date de publication : 1 janvier 2021

Pagination : 191-202

ISSN : 1255-183X

\section{Référence électronique}

Danilo BILATE, «Sade et la valorisation de l'indifférence : le paradoxe de la tolérance libertine »,

Philonsorbonne [En ligne], 15 | 2021, mis en ligne le 03 février 2021, consulté le 08 juin 2021. URL

http://journals.openedition.org/philonsorbonne/1901; DOI : https://doi.org/10.4000/philonsorbonne. 1901

(c) Tous droits réservés 


\title{
Sade et la valorisation de l'indifférence : le paradoxe de la tolérance libertine
}

\author{
Danilo BILATE \\ (Docteur en philosophie de l'Université Paris1 Panthéon -Sorbonne) \\ (Professeur - Université Fédérale Rurale de Rio de Janeiro (UFRRJ) - Brésil)
}

\section{Introduction}

Sade propose une économie, au sens large, une oikonomia libidinale qui établit un commerce entre l'individu et l'objet extérieur dont le but est toujours d'accumuler du plaisir, mais qui dépend, pour se concrétiser, d'un savoir qui mène au contrôle des affects. Cette économie pourrait donc être spécifiée par le génitif «des affects» ou «des passions » ou encore «des sentiments », dans le sens où ledit savoir concerne soit le caractère, soit les dispositions à l'action, soit l'habitude qui en résulte, soit enfin les perturbations caractérologiques qui, bien que passivement subies, sont la cause de toute action humaine. Nous sommes, donc, dans le domaine du pathos et, corrélativement, dans celui de l'éthos et de l'êthos.

Nous pourrions, si nous le voulions, nommer ce savoir « éthique», dans sa forme substantivée, mais ce faisant nous nous plongerions dans une discussion sans doute secondaire ${ }^{1}$. Sade est explicitement un immoraliste, et la prétention à vouloir voir dans sa pensée une éthique se heurte à la difficulté d'établir de façon claire une ordonnance sémantique qui, d'un côté, différencie un tel terme de celui de "morale » et, d'un autre côté, propose une signification qui justifie sa propre utilisation, sans être anachronique. Il nous reste donc à soutenir qu'il y a dans la pensée de Sade une théorie

1. Malgré cela, nous sommes d'accord avec les hypothèses émises par Arlette André, «Sade et l'éthique de l'apathie ", in Mélanges Littéraires François Germain, Dijon, Faculté de lettres et de philosophie de Dijon, 1979, p. 95-104. L'un de nos objectifs est de montrer que la valorisation de l'indifférence est présente partout chez Sade et pas seulement dans l'Histoire de Juliette, roman sur lequel se concentre Arlette André. 
des affects ou une «théorie des passions $»^{2}$, que l'on pourrait qualifier d' « éthique » dans la mesure où elle se réfère à l'action et constitue même un guide - bien que libertin - pour quelqu'un qui veut s'en servir. En effet, s'il n'y avait pas cette théorie, Sade laisserait un vide étourdissant dans son œuvre, car, comme dit son personnage M. de Saint-Florent: «C'est en confondant ainsi tous les sentiments, c'est en n'analysant jamais rien, qu'on s'aveugle sur tout, et qu'on se prive de toutes les jouissances $»^{3}$. De plus, le début de la lettre XXVI d'Aline et Valcour, écrite sous le nom du président de Blamont est péremptoire et décisive : il y parle d'une «étude savante du cœur humain » ou d'un " art des passions [...] qui nous enseigne à mouvoir tour à tour celle qui doit produire un effet désiré ».

Nous ne nions pas ce que dit Le Brun, à savoir que Sade « nous rappelle que l'adhésion, la compréhension sont indissociables du désir [...] [et qu']il dénie la possibilité d'une quelconque autorité pédagogique puisque le désir seul fait autorité ${ }^{4}$. Mais notre hypothèse est que, même sans croire à l'autorité pédagogique du savoir, la compréhension du désir n'a lieu qu'en ayant recours à une théorie. Bien entendu, chez Sade cette théorie ne se présente jamais comme une déontologie et c'est pourquoi Klossowski a écrit que « Sade exerce de façon implicite une critique de la raison normative $»^{5}$. De fait, Sade est un adversaire de la déontologie et de n'importe quelle forme de codification métaphysique des règles praxéologiques pour une raison très simple. Pour lui, les hommes sont nécessairement uniques et singuliers, d'où il s'ensuit que le devoir, en tant que règle, est une aberration qui gomme de façon violente les différences. Pour l'illustrer, lisons, par exemple, un passage de Français, encore un effort :

Ce sera ici une absurdité palpable que de vouloir prescrire des lois universelles ; ce procédé serait aussi ridicule que celui d'un général d'armée qui voudrait que tous ses soldats fussent vêtus d'un habit fait sur la même mesure; c'est une injustice effrayante que d'exiger que des hommes de caractères inégaux se plient à des lois égales : ce qui va à l'un ne va point à l'autre. [...] Il est démontré qu'il y a telle vertu dont la pratique est impossible à certains hommes, comme il $\mathrm{y}$ a tel remède qui ne saurait convenir à tel tempérament ${ }^{6}$.

2. Marco Menin, «Sade et les esprits animaux : du matérialisme électrique au stoïcisme passionné ", in Sylvie Kleiman-Lafon et Micheline Louis-Courvoisier(dir.), Les Esprits animaux $\left(16^{\text {ème }}-21^{\text {ème }}\right.$ siècles). Littérature, histoire, philosophie, Genève, Fondation Hardt, 2016, p. 193. Le même auteur parle, d'ailleurs, d'une « éthique paradoxale » qui « caractérise le libertin » (p. 200). Je remercie Clara Carnicero de Castro pour m'avoir indiqué ce texte du reste, son article «Entre le crime et la sensibilité : les paradoxes du personnage de Clairwil » (in A. Coudreuse et S. Genand (dir.), Sade et les femmes : ailleurs et autrement, Paris, L'Harmattan, 2013, p. 33-44) est très important pour connaître le rôle de l'indifférence dans L'Histoire de Juliette, texte auquel accordent aussi beaucoup d'attention Menin et André (voir la note précédente).

3. Justine ou les malheurs de la vertu, Paris, LGF, 1973, p. 266-267.

4. Annie le Brun, Soudain un bloc d'abîme, Paris, Gallimard, 2010, p. 388.

5. Pierre Klossowski, Le Philosophe scélérat, Paris, Éditions du Seuil, 1967, p. 20.

6. La Philosophie dans le boudoir, « Français, encore un effort », Paris, Gallimard, 1976, p. 208. 
À ce propos, rappelons qu'aussi bien Lacan que Horkheimer et Adorno rapprochent Sade de la pensée kantienne. L'argument central de Lacan pour établir cette relation entre Sade et Kant est que tous deux ont besoin de la loi comme référence morale. Le premier la considérait comme une balise indispensable à la jouissance éprouvée par sa transgression phantasmatique et supposait ainsi implicitement l'existence d'une Loi fondamentale régnant sur ce mécanisme. Le second adoptait le même point de vue d'une Loi fondamentale, mais il recherchait l'obéissance à cette Loi de façon explicite. Il s'agit de «la soumission de Sade à la Loi », étant donné que « la loi et le désir refoulé sont une seule et même chose» et que, par conséquent, «l'apologie du crime ne le pousse qu'à l'aveu détourné de la Loi. L'Être suprême est restauré dans le Maléfice $»^{7}$. Nous pouvons en conclure que même si Lacan peut avoir raison, l'universalisme déontologique que Kant défendra plus tard est si étranger à la pensée sadienne qu'il faudrait peut-être ne pas confondre la structure phantasmatique de l'homme Sade et le contenu de sa pensée philosophique.

En revanche, la lecture faite par Horkheimer et par Adorno souligne la façon dont la raison massifie les gens à travers l'indistinction objectale, ce qui pourrait, selon eux, être observé chez Sade, Kant et Nietzsche. C'est cette massification qui ouvre la voie à la domination violente sur autrui : «L'identité de la domination et de la raison ${ }^{8}$, écrivent-ils. À partir de cette thèse, les auteurs de l'École de Francfort proposent une analyse sociologique, selon laquelle l'indifférence sadienne n'est qu'un exemple plus radical de la tradition « rationaliste " - à cause de la condition bourgeoise de ses représentants. C'est le cas des stoïciens qui, précisément, ont été les premiers à avoir soutenu philosophiquement l'apathie ${ }^{9}$, concept sur lequel nous reviendrons.

De toute façon, nous soutenons que Sade privilégie certains sentiments au détriment d'autres, bouleversant ainsi la table des valeurs traditionnelle, la valorisation de l'indifférence s'inscrivant justement dans ce bouleversement. Cependant, n'oublions pas - au moins en tant qu'observation ponctuelle -, ce que les lecteurs de Sade connaissent bien, à savoir la table inversée des valeurs qu'il propose : il considère comme vices, entre autres, la compassion, la reconnaissance, la pudeur, le remords, la pitié et le sentiment de vengeance. En revanche, ce qui est moins souvent dit, il célèbre l'égoösme, le courage et une certaine forme d'amour ${ }^{10}$ comme étant des vertus.

7. Jacques Lacan, Écrits II, Paris, Gallimard, 1999, « Kant avec Sade », p. 269, 261 et 268, respectivement.

8. Marx Horkheimer \& Theodor W. Adorno, La Dialectique de la Raison, Paris, Gallimard, 1974, « Digression I : Juliette, ou raison et morale », p. 178.

9. «Devant les souffrances des autres, le stoïcisme - qui est la philosophie des bourgeois permet aux privilégiés d'affronter plus facilement la menace qui pèse sur eux » (Ibid., p. 148).

10. Il faut dire que seul l'amour qui se reconnaît comme objectal (et qui, donc, s'appuie sur l'indifférence envers l'altérité) peut être vertueux, dans la mesure où, ainsi, il n'empêche pas l'égoïsme sincère. Comme le dit Dolmancé : «Ce n'est jamais que pour soi qu'il faut aimer les gens ; les aimer pour eux-mêmes n'est qu'une duperie » (La Philosophie dans le boudoir, cinquième dialogue, p. 174). 
L'immoralisme sadien, cette pensée «négative », dans un certain sens « criminelle », étant donné qu'il nie la morale, est déjà bien connu : c'est ce que résume Bataille quand il dit que "détruire » est l'" essence » des ouvrages de Sade ${ }^{11}$. Mais nous oublions souvent que celui-ci a proposé une pensée "positive ", justement à propos des affects et de l'action humaine. Malgré son immoralisme ou son anti-déontologie, il resterait chez lui une pensée qui pourrait nous inspirer des façons d'agir et de vivre, bien que trop excentriques aux yeux d'un grand nombre. La valorisation de l'indifférence n'est donc qu'une facette de cette pensée, mais elle est peut-être la plus importante.

\section{La « vertu» et la nature}

Chez Sade, le critère pour établir la distinction entre vices et vertus est toujours la nature, "cette mère commune ${ }^{12}$. Par conséquent, le véritable crime n'existerait que par rapport aux lois de la nature, comme un écart vis-à-vis du fatum, ce qui est impossible. Écoutons les propos du Comte de Bressac : «Eh ! Non, non, Thérèse, non, la nature ne laisse pas dans nos mains la possibilité des crimes qui troubleraient son économie ${ }^{13}$, ou ceux de Dolmancé, plus directs : «Les crimes sont impossibles à l'homme ${ }^{14}$. Donc, quand nous disons «crime», c'est toujours pour désigner une infraction aux lois humaines, trop humaines.

En ce sens, les « vertus ${ }^{15}$ sont des dispositions ou affects en harmonie avec les lois socialement établies. En revanche, les "vices" sont des dispositions ou affects en désaccord avec ces mêmes lois. Toutefois, si nous suivions la proposition naturaliste de Sade, la véritable vertu dépendrait d'une harmonie entre le corps et les impulsions de la nature et le véritable vice serait la disposition antinaturelle, à savoir la résistance corporelle à ces impulsions-là. C'est cela que Beauvoir résume dans la phrase suivante : «[...] du credo généralement accepté "La Nature est bonne, suivons-la", Sade en rejetant le premier point conserve paradoxalement le second ${ }^{16}$.

C'est en ce sens que nous devons comprendre les enseignements des instituteurs philosophes-sexuels sadiens, comme, par exemple, ceux que Dolmancé dispense à Eugénie. Si en prenant la nature comme critère, la

11. Georges Bataille, La Littérature et le mal, Paris, Gallimard, 1957, p. 82.

12. La Philosophie dans le boudoir, « Français, encore un effort», op. cit., p. 209.

13. Justine ou les malheurs de la vertu, op. cit., p. 104.

14. La Philosophie dans le boudoir, septième dialogue, op. cit., p. 280.

15. Nous mettons les guillemets ici pour souligner «le vide et le néant de la vertu », (selon le Duc de Blangis, Les 120 journées de Sodome, Introduction, Paris, Éditions 10/18, 1975, p. 22), en donnant au mot « vertu » le sens ordinaire de quelque chose d'universel et d'éternel. Le même usage des guillemets vaut pour le mot " vice».

16. Simone de Beauvoir, Faut-il brûler Sade?, Paris, Gallimard, 1955, p. 70. 
notion commune de «vertu » est fausse, nous devons conclure que celle-ci est beaucoup moins importante que la satisfaction corporelle. Comme le dit Dolmancé : «Va, la vertu n'est qu'une chimère, dont le culte ne consiste qu'en des immolations perpétuelles [...]. Une seule goutte de foutre éjaculée de ce membre, Eugénie, m'est plus précieuse que les actes les plus sublimes d'une vertu que je méprise ${ }^{17}$. Comme résistance aux impulsions naturelles, la «vertu» est méprisable et c'est pourquoi l'instituteur demande à sa disciple : «Révérerez-vous l'obligation de combattre tous les mouvements de la nature ? ${ }^{18}$.

Constater que la nature est un critère ou une référence menant à une réévaluation de la table des vertus entraîne chez le philosophe une recherche physique sur la nature et sur le corps. Cette double investigation n'étant qu'une, car le corps est une partie de la nature et obéit à ses impulsions, toutes les conclusions auxquelles nous parvenons permettent de juxtaposer ontologie et éthique. En d'autres termes, la théorie des affects ne serait qu'une résultante des axiomes ontologiques et la nouvelle table de vertus devrait s'adapter aux lois naturelles.

\section{L'amoralité de la nature et l'innocence fondamentale de l'homme}

Sade caractérise la nature comme une force matérielle incessante hors de laquelle rien n'existe, un mouvement perpétuel de création et donc aussi un devenir destructif qui permet cette création. Par conséquent, toutes les variations matérielles sont nécessaires et rien n'offense la nature. Le devenir est donc innocent. Comme l'affirme le Comte de Bressac, «Toute forme est égale aux yeux de la nature; rien ne se perd dans le creuset immense où ses variations s'exécutent; toutes les portions de matières qui y tombent en rejaillissent incessamment sous d'autres figures, et quels que soient nos procédés sur cela, aucune ne l'outrage sans doute, aucun ne saurait l'offenser $»^{19}$. Innocente, en tant que devenir nécessaire, la nature est pardelà le bien et le mal. En conséquence, les vertus et les vices sont tout aussi incontournables qu'acceptables. Étant donné qu' « un seul moteur agit dans l'univers, et ce moteur, c'est la nature », selon Dolmancé, « la nature, qui, pour le parfait maintien des lois de son équilibre, a tantôt besoin de vices et tantôt besoin de vertus, nous inspire tour à tour le mouvement qui lui est nécessaire $»^{20}$.

17. La Philosophie dans le boudoir, troisième dialogue, op. cit., p. 67.

18. La Philosophie dans le boudoir, troisième dialogue, op. cit., p. 76.

19. Justine ou les malheurs de la vertu, op. cit., p. 102.

20. La Philosophie dans le boudoir, septième dialogue, op. cit., p. 279. 
Cependant, ce devenir destructif et créatif, innocent et nécessaire, obéit à des lois établies dont la plus manifeste est l'effort d'auto-conservation : « Nous n'aspirons qu'à vivre, et c'est la première de toutes les lois », dit Brigandos $^{21}$. Il ne s'agit ici ni du conatus spinoziste, ni de la volonté de puissance nietzschéenne, mais d'une pulsion égoïste qui entraîne l'individu dans la quête effrénée du plaisir. Étant inconditionnelle, elle est une force amorale et immorale qu'aucun obstacle n'arrête et surtout pas l'empathie envers la douleur d'autrui. L'altérité est chez Sade toujours objectale et l'objet est une source potentielle de plaisir. Écoutons encore une fois Dolmancé : «Ah ! Croyons-le, croyons-le, Eugénie, la nature, notre mère à tous, ne nous parle jamais que de nous ; rien n'est égoïste comme sa voix, et ce que nous y reconnaissons de plus clair est l'immuable et saint conseil qu'elle nous donne de nous délecter, n'importe aux dépens de qui $»^{22}$. Ainsi, il faut en conclure que tout est permis et que commettre un crime ou qu'être vicieux fait partie de la nature autant qu'être vertueux : «Eh, que m'importe le crime, répond Durcet, pourvu que je me délecte. Le crime est un mode de la nature, une manière dont elle meut l'homme. Pourquoi ne voulez-vous pas que je me laisse mouvoir aussi bien par elle en ce sens-là que par celui de la vertu? Elle a besoin de l'un et de l'autre, et je la sers aussi bien dans l'un que dans l'autre $»^{23}$. L'homme est donc une partie constitutive du devenir, une fraction de la totalité, un fait lui aussi, puisqu'il est une partie intégrante du continuum également nécessaire. L'individu ne peut servir que la nature, attaché à elle dans la mesure où il la constitue.

Cela dit, nous pouvons néanmoins poser encore une fois la question de Simone de Beauvoir: "Pourquoi des chemins qui s'ouvrent devant lui Sade a-t-il donc choisi celui qui par l'imitation de la nature le conduit au crime ? $»^{24}$. À vrai dire, la réponse a déjà été donnée ${ }^{25}$. Si la «vertu» fait partie de la nature tout comme le "vice», seul ce dernier suit la loi fondamentale de l'égoïsme. Autrement dit, la valorisation sadienne du crime est le refus de la loi humaine, refus de la table des valeurs actuelle, refus dû au constat que la culture morale est antinaturelle. La vertu et le vice font partie de la nature parce que celle-ci est une totalité qui englobe donc toutes les choses indistinctement. N'étant qu'une de ces choses, l'homme, du point de vue de la nature, est aussi innocent qu'elle. Il s'agit ici d'une des assertions les plus fréquentes chez Sade :

21. Aline et Valcour, Lettre XXXVIII, Paris, LGF, 1994, p. 513.

22. La Philosophie dans le boudoir, troisième dialogue, p. 129. Voir aussi l'explication sur « l'égoïsme intégral » de Maurice Blanchot (Lautréamont et Sade, Paris, Minuit, 1963, p. 19).

23. Les 120 journées de Sodome, I, quinzième journée, op. cit., p. 235.

24. Simone de Beauvoir, op. cit., p. 72.

25. Par Simone de Beauvoir elle-même, sans qu'apparemment elle en ait été satisfaite : « Ce qui interdit à la liberté de l'individu d'opter pour le bien, c'est que celui-ci n'existe ni dans le ciel vide, ni sur la terre injuste, ni même dans un horizon idéal : il n'est nulle part » (Simone de Beauvoir, op. cit., p. 79). Son insatisfaction, s'il en est, s'explique par le fait qu'elle ne reconnaît pas l'impulsion naturelle de l'égoïsme comme étant un critère axiologique pour Sade. 
Voilà l'essentiel et voilà ce que je promets. Des vertus, on ne s'en fait pas, et on n'est pas plus le maître d'adopter dans ces choses-là tel ou tel goût, qu'on n'est le maître de devenir droit quand on est né tortu, pas plus le maître d'adopter en fait de système telle ou telle opinion, que de se faire brun quand on est né roux. Voilà mon éternelle philosophie, et jamais je n'en sortirai ${ }^{26}$.

En bref, au sens ontologique de la liberté, l'homme n'est point libre. D'ailleurs, dans le domaine des valeurs tout est relatif. Les mœurs sont marquées géographiquement et historiquement, arbitraires puisqu'il s'agit de conventions culturelles. Il n'existe donc pas de chose bonne en soi : «Apprenez que c'est le point où l'on est qui rend une chose bonne ou mauvaise, et non pas la chose en elle-même. On guérit les paysans russes de la fièvre avec de l'arsenic; l'estomac d'une jolie femme ne s'arrangerait pourtant pas de ce remède-là. Voilà donc la preuve que tout est relatif $\gg^{27}$.

Du point de vue de la nature, le seul critère axiologique significatif pour Sade, personne n'est coupable de rien. Si l'homme n'est pas libre, le concept de «faute» s'évanouit. C'est le sens des questions posées par Duclos dans les 120 journées: "Mais est-ce ma faute? N'est-ce pas de la nature que nous tenons nos vices ou nos perfections, et puis-je adoucir ce cœur qu'elle a fait insensible ? $»^{28}$. Autrement dit, la nature est indifférente à toute action humaine. D'après Sarmiento : "Toute manière de se conduire, absolument indifférente en elle-même, devient bonne ou mauvaise en raison du pays qui la juge $»^{29}$. L'indifférence caractéristique de la nature signifie ici qu'elle ne différencie rien et ne peut rien différencier, car, pour elle, tout est ou fait partie d'elle-même.

\section{De l'indifférence de la nature vers l'indifférence de l'individu}

Le mot d' « indifférence » et ses variations sont des signifiants très importants et courants chez Sade et nous pouvons même affirmer que dans sa théorie des affects l'indifférence est centrale. Ce n'est pas un hasard si Thérèse dit de $M$. de Gernande, le Comte, que «telle est la fatale indifférence qui caractérise, mieux que tout, l'âme d'un véritable libertin ${ }^{30}$. Et cela parce que, comme le déclare Eugénie, «rien n'est aussi indifférent sur la terre que d'y commettre le bien ou le mal ${ }^{31}$. C'est parce que la nature est axiologiquement indifférente, parce qu'elle ne différencie pas ses

26. Lettre à sa femme fin août 1782 ; Lettres à sa femme, Choix de Marc Buffat, Arles, Actes Sud, 1997, p. 334.

27. Lettre à sa femme en juillet 1783, op. cit., p. 390.

28. Les 120 journées de Sodome, douzième journée, op. cit., p. 202.

29. Aline et Valcour, Lettre XXXV, op. cit., p. 225.

30. Justine ou les malheurs de la vertu, op. cit., p. 247.

31. La Philosophie dans le boudoir, troisième dialogue, op. cit., p. 79. 
propres parties constitutives, que l'homme qui reconnaît ce fait et qui prend la nature comme critère ne peut pas croire aux valeurs tout simplement humaines.

Pourrions-nous voir ici une contradiction qui partirait de la découverte de l'indifférence fondamentale de la nature pour s'en saisir afin d'établir un guide vers le plaisir de réaliser ses désirs? Il semble que Sade joue avec cette contradiction sans s'en préoccuper : «[...] Il est indifférent au plan général, que tel ou tel soit bon ou méchant de préférence, que si le malheur persécute la vertu et que la prospérité accompagne le crime, les choses étant égales aux vues de la Nature, il vaut infiniment mieux prendre parti parmi les méchants qui prospèrent $\gg^{32}$, ou enfin, comme le résume Dolmancé : «Pourvu que je sois heureux, le reste m'est absolument égal ${ }^{33}$. Devant la découverte de la neutralité axiologique constitutive du devenir, le seul conseil à donner est de suivre l'impulsion la plus forte qui nous meut, à savoir l'impulsion de chercher toujours, de façon égoïste, le maximum de plaisir.

Il y a ici une forme d'effacement moral de l'individu qui, en se reconnaissant comme n'étant qu'une partie constitutive du tout, comprend qu'il faut se soumettre au fatum. Qu'il s'agisse de se soumettre par amour ou par courage, ce qui est certain c'est que Sade ne propose ni immobilisme ni quiétude. Certes, nous, les hommes, nous ne sommes pas ontologiquement libres, car nous agissons toujours selon ce que la nature nous inspire, autrement dit, d'après les impulsions naturelles qui nous constituent. C'est pourquoi Bersac affirme: "Mais malheur au sophiste qui conclurait de là, qu'il doit, ou adopter le vice, ou se consoler de ne pas être vertueux, puisqu'il accomplit les lois de la nature $»^{34}$.

Curieusement, Sade parle souvent d'un stoïcisme et emploie même parfois le mot "apathie », mais il nie péremptoirement l'idée d'équilibre ou d'harmonie. Cela est flagrant, par exemple, quand il dit, par la bouche de Dolmancé : «Ah ! Croyez, Eugénie, croyez que les plaisirs qui naissent de l'apathie valent bien ceux que la sensibilité vous donne; celle-ci ne sait qu'atteindre dans un sens le cœur que l'autre chatouille et bouleverse de toutes parts ${ }^{35}$. Une « apathie » qui chatouille et bouleverse est une contradiction dans les termes. En l'employant ici, Sade donne une nouvelle signification au signifiant. Ce n'est pas au sens étymologique, c'est-à-dire comme négation du pathos ou négation des affects, mais bien le contraire. Donc, ce n'est pas vraiment l'apatheia des stoïciens, mais plutôt l'expérimentation individuelle de l'indifférence de la nature. Les paroles du Comte de Bressac exemplifient bien cette juxtaposition éthico-ontologique :

32. Selon le narrateur dans Justine ou les malheurs de la vertu, op. cit., p. 30.

33. La Philosophie dans le boudoir, cinquième dialogue, op. cit., p. 258.

34. Aline et Valcour, Lettre XXXVIII, op. cit., p. 626.

35. La Philosophie dans le boudoir, cinquième dialogue, op. cit., p. 257. En analysant ce passage Marco Menin affirme que « la maîtrise de soi, généralement considérée comme le but ultime du cheminement de la sagesse spirituelle qui pousse l'individu à se soumettre à un ordre supérieur, devient chez le Divin Marquis le moyen d'accéder à la pleine expression, égoïste et complètement terrestre, de la dimension passionnelle » (op. cit., p. 204). 
L'être qui lui ressemble le mieux [à la nature], et par conséquent l'être le plus parfait, sera donc nécessairement celui, dont l'agitation la plus active deviendra la cause de beaucoup de crimes, tandis, je le répète, que l'être inactif ou indolent, c'est-à-dire l'être vertueux, doit être à ses regards le moins parfait sans doute, puisqu'il ne tend qu'à l'apathie, qu'à la tranquillité qui replongerait incessamment tout dans le chaos, si son ascendant l'emportait ${ }^{36}$.

La vraie apathie, celle que critique le Comte de Bressac, c'est la tranquillité ou la tempérance des vertueux. Presque toujours, les héros sadiens font tout par plaisir, un plaisir qui doit être le plus intense possible, c'est-à-dire qu'ils cherchent à éprouver les chatouillements et bouleversements physiques dont parlait Dolmancé. Mais les scélérats, comme l'est la nature et comme le propose la théorie sadienne des sentiments, sont indifférents aux mœurs et aux lois, en un mot, indifférents à toute forme d'altérité. Par conséquent, le pathos qu'ils n'éprouvent pas est seulement la pitié et c'est uniquement en ce sens qu'ils peuvent être considérés comme « apathiques ». En bref, comme le rappelle Castro : "Les termes "sensibilité" et "passion" sont ambivalents : ils ont une connotation négative lorsqu'ils révèlent une identification à autrui, mais positive s'ils sont rapportés à la recherche égoïste du plaisir. Le but de l'apathie serait donc d'émousser la sensibilité morale, pour exalter ensuite la sensibilité physique $»^{37}$.

Cependant, les libertins sadiens ne sont bien sûr pas indifférents au plaisir. Ce genre de stoïcisme paradoxal ${ }^{38}$ proposé par Sade consiste à savoir se soumettre à la nature, supporter courageusement le destin, subir tout avec calme et froideur, mais toujours en recherchant le plaisir, en obéissant donc à la principale loi de la nature. Pour le conquérir, le scélérat apprend à opérer ce que Binoche a appelé une «transmutation des affects » ${ }^{39}$, c'est-à-dire, la capacité, par indifférence, à ne jamais se chagriner, en transformant la souffrance en plaisir. D'après Léonore, «il ne s'agirait dans ce cas que d'avoir de la philosophie pour recevoir des impressions très singulières, et par conséquent pour étendre étonnamment la sphère de ses jouissances $\gg{ }^{40}$. Lisons la description que fait Julie de l'assertion de M. de Blamont :

36. Justine ou les malheurs de la vertu, op. cit., p. 104.

37. Clara Carnicero de Castro, op. cit., p. 38.

38. Voir l'explication sur « le noir stoïcisme de Sade» ou « la paradoxale parenté du sadisme et du stoïcisme », selon Beauvoir (op. cit., p. 85-86) et l'« insensibilité stoïque » dont parle Blanchot (Lautréamont et Sade, op. cit., p. 31).

39. Bertrand Binoche, Sade ou l'institutionnalisation de l'écart, Québec, PUL, 2007, p. 32. L'auteur y affirme que Blanchot fournit une excellente explication à ce propos : «Pour Sade, l'homme souverain est inaccessible au mal, parce que personne ne peut lui faire de mal ; il est l'homme de toutes les passions, et ses passions se plaisent à tout " (Maurice Blanchot, Lautréamont et Sade, op. cit., p. 28; voir aussi les pages 23-24, 27-30). La clé d'interprétation de Blanchot se trouve, sans aucun doute, dans l'assertion suivante: «Personne ne peut lui porter atteinte [à “l'homme intégral”, le héros sadien], rien n'aliène son pouvoir d'être soi et de jouir de soi » (p. 30).

40. Aline et Valcour, Lettre XXXIX, op. cit., p. 646. 
Là-dessus, M. le président répondit que la plus haute de toutes les folies était de se chagriner, qu'il fallait savoir monter son âme à une sorte de stoïcisme, qui nous fît regarder avec indifférence tous les événements de la vie ; que pour lui, loin de s'affliger de rien, il se réjouissait de tout [...] et qu'avec ce système on parvenait à changer en roses toutes les épines de la vie. [...] Et il assura mademoiselle qu'elle serait toujours malheureuse tant qu'elle n'adopterait pas cette prudente philosophie ${ }^{41}$.

Ces paroles de M. de Blamont sont très claires : «Loin de s'affliger de rien, il se réjouissait de tout ». L'indifférence n'est pas donc une négation neutre du pathos, une apathie donc, mais une attitude affective qui fait que l'individu est capable de se réjouir de tout, sans distinction. Et Blamont lui-même déclare : «Or l'homme le plus malheureux, sans doute, est celui qui, ne sachant pas l'art de voltiger également sur tous les plaisirs... de les effleurer tous sans s'appesantir sur aucun, s'est fait d'une sorte de goûts une si forte habitude, qu'il ne peut plus y renoncer sans douleur. Usons de tout et ne nous attachons à rien $[\ldots] »{ }^{42}$. « Ne s'attacher à rien » est l'enseignement que Sade nous dispense à partir du moment où il a constaté l'indifférence de la nature. Là, justement, on ne différencie pas l'altérité de la condition d'objet, de ce qui peut être utilisé pour l'obtention de plaisir. L'autre ne se détache de la totalité du réel que comme un instrument destiné à la jouissance $^{43}$. L'individu ne peut alors que calculer économiquement pour administrer stratégiquement ce qu'il peut dans le jeu constant d'action, ce corps étant animé sans cesse par la recherche du plaisir. Telle est la signification de la longue et importante déclaration de Dubois :

Il ne faut jamais calculer les choses que par la relation qu'elles ont avec nos intérêts. La cessation de l'existence de chacun des êtres sacrifiés est nulle par rapport à nous. Assurément nous ne donnerions pas une obole pour que ces individus-là fussent en vie ou dans le tombeau; conséquemment si le plus petit intérêt s'offre à nous avec l'un de ces cas, nous devons sans aucun remords le déterminer de préférence en notre faveur; car dans une chose totalement indifférente, nous devons, si nous sommes sages et maîtres de la chose, la faire indubitablement tourner du côté où elle nous est profitable, abstraction faite de tout ce que peut y perdre l'adversaire ; parce qu'il n'y a aucune proportion raisonnable entre ce qui nous touche, et ce qui touche les autres; nous sentons l'un physiquement, l'autre n'arrive que moralement à nous, et les sensations morales sont trompeuses; il n'y a de vrai que les sensations physiques ${ }^{44}$.

41. Aline et Valcour, Lettre LXVIII, op. cit., p. 770.

42. Aline et Valcour, Lettre LXVII, op. cit., p. 756.

43. C'est en ce sens que Jean Deprun considère l' " isolisme » comme l'un des trois aspects à côté de l'intensivisme et de l'antiphysisme - de l'«éthique sadienne » (« Sade et le rationalisme des Lumières », in Raison présente, $\mathrm{n}^{\mathrm{o}} 3$ 3, 1967, p. 81).

44. Justine ou les malheurs de la vertu, op. cit., p. 69. 


\section{Conclusion : l’indifférence comme tolérance libertine}

Nous découvrons ici avec étonnement l'apologie que Sade fait de la tolérance. Cet auteur, vu souvent comme libertin, maudit et criminel, proposerait la tolérance comme un sentiment à cultiver. Et cela va de soi. $\mathrm{Si}$ la nature est indifférente et si le libertin doit être aussi indifférent qu'elle, il peut éprouver une sorte de tolérance précisément parce qu'il est indifférent. En ce sens, nous sommes d'accord avec Delon, quand il affirme que « le refus intéressé de condamner le goût d'autrui devient conscience de la diversité, nécessité de la connaître, de la répertorier, voire de la protéger. L'irréductibilité à une quelconque norme laisse place à un principe commun à toutes les manies, un raffinement, un tact, qui pousse chacun à son libertinage propre $\gg{ }^{45}$. En revanche, nous ne sommes pas tout à fait d'accord avec lui quand il conclut que «le principe de tolérance se renverse en revendication élitiste. Le désir ne veut pas être toléré, il prétend s'imposer », ce qui signifierait «le refus de toute singularité au nom d'une radicalité toujours approfondie ${ }^{46}$. Son hypothèse est que la tolérance qui existe chez un individu plus immature serait ultérieurement surmontée par le libertin plus expérimenté, lorsqu'il reconnaît la « radicalité » du désir qui aboutit même à la destruction d'autrui. Il nous semble que l'irréductibilité à une norme est une prise de position constante chez Sade, ce qui mène à une inévitabilité de la tolérance. Et le fait que le désir s'impose toujours ne nie pas la possibilité de tolérer le désir d'autrui. La clé de la compréhension, semble-t-il, consiste à comprendre que «tolérance» n'est pas la même chose que pitié ou compassion. Autrement dit, le libertin sadien peut désirer éliminer celui qui désire d'une autre manière - celui qui désire conformément aux lois sociales, par exemple -, ce qui est très fréquent chez Sade.

Étant donné que l'autre n'est qu'un objet pour le scélérat, le criminel sadien n'éprouve ni ressentiment, ni jalousie, ni sentiment de vengeance, ni aucun autre affect dépendant d'une relation d'altérité personnalisée, puisque le seul intérêt admis est celui de jouir de l'objet. Même si le criminel sadien est toujours ou presque toujours agressif, cela n'est jamais dû à une haine née de la rancune, mais uniquement à l'envie d'avoir du plaisir. Cette recherche aveugle et sauvage de plaisir dépersonnalise autrui, en le rabaissant à la condition objectale. Ainsi, la tolérance libertine qui ne repose pas sur la pitié est paradoxale : elle tolère le désir d'autrui seulement dans la mesure où celui-ci est dépersonnalisé. C'est pourquoi le chevalier de $L a$ Philosophie dans le boudoir déclare : "L'homme est-il maître de ses goûts ? Il faut plaindre ceux qui en ont de singuliers, mais ne les insulter jamais : leur tort est celui de la nature ${ }^{47}$. L'individu ne peut donc pas être insulté à

45. Michel Delon, « La tolérance en amour : de Sade à Fourier », in Études littéraires, vol. 32, $\mathrm{n}^{\circ} 1-2,2000$, p. 222.

46. Ibidem, p. 223 et p. 224.

47. La Philosophie dans le boudoir, premier dialogue, op. cit., p. 42-43. 
cause de ses désirs, car il n'est qu'une partie constitutive de la nature. Et il ne s'agit pas ici d'un passage fortuit chez Sade, comme le démontre l'affreux duc des 120 journées : «[...] chacun a sa manie; nous ne devons jamais ni blâmer, ni nous étonner de celle de personne $»^{48}$. Les propos du monstrueux Clément vont dans le même sens :

Encore une fois dans l'un et l'autre cas, sa singularité est le résultat de ses organes : est-ce sa faute, si ce qui vous affecte est nul pour lui, ou s'il n'est ému que de ce qui vous répugne? Quel est l'homme qui ne réformerait pas à l'instant ses goûts, ses affections, ses penchants sur le plan général, et qui n'aimerait pas mieux être comme tout le monde, que de se singulariser s'il en était le maître ? Il y a l'intolérance la plus stupide et la plus barbare à vouloir sévir contre un tel homme ${ }^{49}$.

Il est vrai que Sade se sert de ces passages pour défendre la position libertine contre l'intolérance moraliste, et peut-être son argumentation comporte-t-elle une certaine dose d'ironie, mais elle peut aussi être utilisée en faveur de toute singularité désirante. Il est certain que la théorie sadienne des affects est très immoraliste, c'est-à-dire qu'elle va totalement à l'encontre des morales existantes. Toutefois, ce que Lacan a pressenti se confirme d'une autre façon, à savoir par le biais de ce que dit Sade de la tolérance. La Loi demeure présente, comme une exigence de respect vis-àvis des singularités humaines, conséquence contradictoire du discours rationalisé adaptable à l'exigence de jouissance. Ces deux impératifs, bien que contradictoires, rapprochent Sade de la forme autoritaire de la déontologie. Mais tous deux acceptent la singularité de chaque désir individuel, ce qui s'oppose au caractère universel de tout impératif. La contradiction est ici insurmontable, et c'est pourquoi le libertin ne peut que signaler de façon simplement descriptive et presque neutre l'absurde de l'intolérance.

Il est certain que le Sade immoraliste qui suggère de détruire l'ensemble des valeurs dominantes est bien connu. Mais, à cause de ce masque qu'on lui a mis - par-delà le masque de l'écrivain pornographique et de l'apologiste de la violence - et parce que peu de gens cherchent à découvrir ce que cache ce masque, l'autre Sade demeure inconnu: celui qui inverse le schéma des valeurs dominantes pour proposer une table des vertus dont cette forme étrange, puisque libertine et non compassionnelle, de tolérance par indifférence.

48. Les 120 journées de Sodome, I, dix-huitième journée, op. cit., p. 263.

49. Justine ou les malheurs de la vertu, op. cit., p. 200. 